Research Paper

\title{
Risk of immune-related diarrhea with PD-1/PD-L1 inhibitors in different cancer types and treatment regimens
}

\author{
Lei Zhao, Jing Yu, Jing Wang, Huihui Li, Juanjuan Che, Bangwei Cao ${ }^{\varpi}$ \\ Cancer center, Beijing Friendship Hospital, Capital Medical University, Beijing 100050, China \\ $\triangle$ Corresponding author: Dr. Bangwei Cao, Cancer center, Beijing Friendship Hospital, Capital Medical University, 95 Yong An Road, Xicheng District, Beijing \\ 100050, China.TEL: +86-010-63139321,Fax: +86-010-63139321, E-mail address: oncology@ccmu.edu.cn. \\ (C) The author(s). This is an open access article distributed under the terms of the Creative Commons Attribution License (https://creativecommons.org/licenses/by/4.0/). \\ See http://ivyspring.com/terms for full terms and conditions.
}

Received: 2019.01.01; Accepted: 2019.06.25; Published: 2020.01.01

\begin{abstract}
Objective: To compare the incidence and severity of diarrhea among different tumor types and treatment regimens, and also compared with CTLA-4 inhibitors in randomized controlled trials.

Methods: MEDLINE, PMC database and EMBASE were retrieved until December 2018. Studies were eligible if they were randomized controlled trials and included participants undergoing PD-1/PD-L1 inhibitors for cancer, measured a treatment side effect of diarrhea, and reported quantitative data. The risks of diarrhea in PD-1/PD-LI inhibitors were compared among different treatment regimens.

Results: Totally 21 studies involving 11554 patients were included for meta-analysis. For all-grade diarrhea, the risk after the PD-1/PD-L1 inhibitors plus CTLA-4 inhibitor combination was 1.90 times significantly higher than that of monotherapy, and the risk was 0.69 and 0.60 times significantly lower than that of monotherapy compared with chemotherapy and ipilimumab. The incidence of diarrhea was not significantly different between PD-1/PD-Ll inhibitor monotherapy versus placebo or between low-doses versus high-doses. For high-grade (grade $\geq 3$ ) diarrhea, the risk after the PD-1/PD-L1 inhibitors plus CTLA-4 inhibitor combination was 3.29 times significantly higher than that of monotherapy, the risk in PD-1/PD-Ll inhibitors monotherapy was 0.50 and 0.38 times significantly lower than chemotherapy and ipilimumab respectively. No significant difference was found in the incidence of diarrhea between PD-1/PD-L1 inhibitor monotherapy versus placebo or between low-doses versus high-doses whether in all-grade or high-grade group.

Conclusions: PD-1/PD-L1 inhibitors have a lower risk of developing diarrhea than chemotherapy and CTLA-4 inhibitor. There is no direct relationship between the dose of PD-1/PD-L1 inhibitors and the risk of developing diarrhea.
\end{abstract}

Key words: Cancer; Diarrhea; Randomized controlled trials; PD-1/PD-L1 inhibitors; CTLA-4 inhibitor; Chemotherapy

\section{Introduction}

Increasing evidence proves the significant efficacy of immune checkpoint inhibitors (ICIs) in treatment of advanced cancers [1-4]. ICIs targeting the programmed cell death protein 1 / programmed death ligand 1 (PD-1/PD-L1) pathway significantly improve the progression-free survival and overall survival compared with standard chemotherapy, so
PD-1/PDL1 antibodies are currently approved for treatment of various malignancies [5-11]. Since the anti-PD-1 antibody pembrolizumab was approved in September 2014 for treatment of advanced melanoma, the clinical development of PD-1/PD-L1 inhibitors as anticancer drugs has been widely expanded. Currently, the Food and Drug Administration has 
approved PD-1/PD-L1 inhibitors for treatment of 9 types of cancers. For instance, pembrolizumab can be used to treat melanoma [2, 12-14], non-small cell lung cancer (NSCLC) [7, 15-19], head and neck squamous cell carcinoma (HNSCC) [20], Hodgkin's lymphoma [21], urothelial cancer [22, 23] and gastric cancer [24]. Anti-PD-1 antibody nivolumab is recommended for treating melanoma [11, 25], renal cell carcinoma (RCC)[26], Hodgkin's lymphoma [27, 28], urine epidermal cancer [29], MSI-H colon cancer [30] and hepatocellular carcinoma [31]. Anti-PD-L1 antibody atezolizumab is suggested for treatment of urothelial cancer $[22,32]$ and NSCLC $[6,33]$, and anti-PD-L1 antibodies avelumab and durvalumab can be used to treat urothelial cancer[34, 35]. Compared with cytotoxic chemotherapy, ICIs have unique toxicity based on their action mechanism, which is considered to be immune-related adverse event (IRAE) [36-39]. Inhibiting the PD-1/PD-L1 pathway may lead to autoimmune toxicity, some of which may be severe or even life- threatening [36, 40].

Diarrhea is a common side effect of cancer treatment that, in severe cases, can lead to death or to patients having to stop lifesaving treatment because often there are no effective therapies to control the diarrhea. Diarrhea in cancer patients can quickly lead to life-threatening consequences such as dehydration, electrolyte imbalance, shock, etc. Compared to chemotherapy-related diarrhea the immunological preparation of PD-1/PD-L1 is prone to cause autoimmune digestive diseases such as ulcerative colitis, and may also cause side effects of diarrhea.

Given the clinical efficacy evidence for a wide spectrum of tumor types, the PD-1 ICI therapy is expected to be increasingly used by oncologists as a monotherapy or in combination with other drugs. Therefore, physicians in cancer immunotherapy must be familiar with the pathogenesis of diarrhea in different tumors and different treatment regimens, and provide useful information to optimize the management of this toxicity. At present, there is no complete description about the clinical experience of anti-PD-1/PD-L1-associated diarrhea patients, or about the management and outcome of this toxicity. Therefore, we conducted a meta-analysis of PD-1 inhibitors in cancer patients and compared the incidence and severity of diarrhea among different tumor types, different treatment regimens.

\section{Methods}

\subsection{Literature selection and data extraction}

Two researchers (Lei Zhao and Huihui Li) independently reviewed the databases Medline, PMC database and EMBASE to select potential relevant articles. Any discrepancy between them was resolved by consensus. The following medical subject heading terms were used: PD-1, PDL1, CD274, programmed death receptor 1 , programmed death receptor ligand, immune checkpoint inhibitor, nivolumab, BMS936558, pembrolizumab, MK-3475, MPDL3280A, atezolizumab, avelumab, MSB0010718C, durvalumab, and diarrhea. The databases were searched from the inception until December, 2018.

The inclusion criteria were: (a) phase I, II and III trials in cancer patients; (b) random assignment of participants to single PD-1/PD-L1 inhibitor treatment or other control therapy (e.g. ipilimumab, placebo); (c) reporting diarrhea events or event rate and sample size for any all-grade or high-grade $(\geq 3)$ adverse events;(d) random controlled trial.

The following information was extracted by two independent reviewers (Lei Zhao and Huihui Li) from the included studies: first author, publication year, study name, clinical trial registration number, total number of patients, mean age, trial phase, treatment plan, tumor type, primary inclusion criteria, and numbers of patients with all grades and high-grade treatment-related diarrhea. The treatment regimens were classified as PD-1/PD-L1 inhibitor monotherapy, PD-1/PD-L1 inhibitor plus CTLA-4 inhibitor ipilimumab, chemotherapy, placebo, and ipilimumab. According to the different doses, monotherapy was divided into low-dose group and high-dose group.

\subsection{Quality assessment}

The two reviewers (Lei Zhao, Huihui Li) used the Jadad scoring method[41] to evaluate the quality of each included study from randomized ( 0 or 1$)$, double-blind $(0,1$ or 2$)$, recorded loss of follow-up and/or exit ( 0 or 1 ) and assign hidden ( 0 or 1$)$. A score $\geq 3$ indicates high quality.

\subsection{Statistical analysis}

Meta-analysis for statistical analysis was performed using Stata12.1 (Stata Corp, College Station, TX, USA). Heterogeneity was analyzed by Q test, and $\mathrm{I}^{2}<25 \%, 25 \%-75 \%$, and $>75 \%$ indicate mild, moderate and significant heterogeneity, respectively. In case of insignificant heterogeneity between studies indicated as $P>0.05$, a fixed effect model was used; otherwise a random effect model was used. The incidence of diarrhea was evaluated by relative risk (RR) and 95\% confidence interval (CI), and the analysis results were represented by forest maps. Two-tailed $p<0.05$ was considered significant. This meta-analysis has been registered on the PROSPERO website (Registration Number: CRD42018111834). 


\section{Results}

The database search initially returned 4021 studies. After screening and eligibility assessment, a total of 21 randomized controlled trials (RCTs, $\mathrm{n}=11554$ patients) were identified for meta- analysis. ICIs tested in these studies included nivolumab $(n=13$ studies), pembrolizumab $(\mathrm{n}=6)$, avelumab $(\mathrm{n}=1)$ and durvalumab $(\mathrm{n}=1)$. Tumor types tested included NSCLC ( $n=7$ studies), melanoma $(n=10)$, HNSCC $(n=1)$, Renal Cell Carcinoma (RCC) $(n=1)$, gastric cancer $(n=1)$ and small cell lung cancer (SCLC) $(n=1)$. According to the clinical staging, 15, 5 and 1 of the 21 RCTs were at phase 3, 2 and 1, respectively (Table 1, Figure 1).

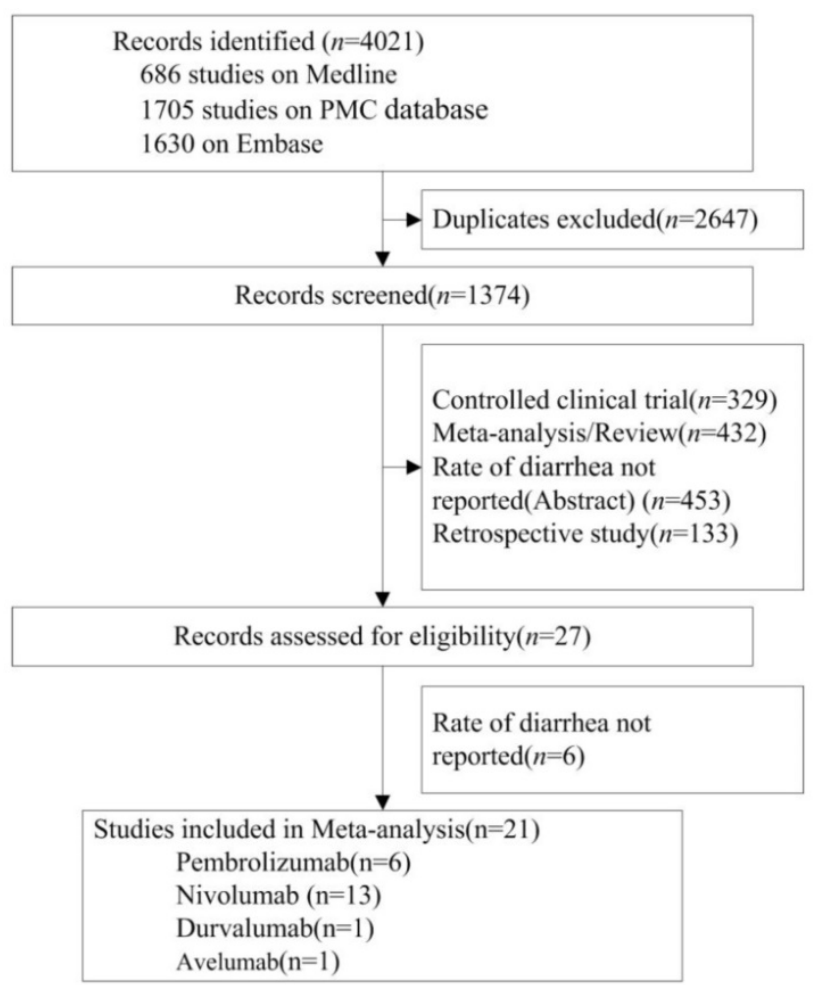

Figure 1. Flow Diagram of Study Inclusion.

\subsection{Risks of diarrhea among different treatment regimens}

\section{All-grade diarrhea}

The risks of all-grade diarrhea in PD-1/PD-L1 inhibitors were compared among different treatment regimens: PD-1/PD-L1 inhibitor monotherapy versus PD-1/PD-L1 inhibitor plus ipilimumab, versus chemotherapy, versus placebo, versus ipilimumab, and high-dose versus low-dose in the 20 studies (Figure 2).

\section{PD-1/PD-L1 inhibitor monotherapy versus placebo}

Two RCTs [42, 43] compared PD-1/PD-L1 inhibitor monotherapy and placebo $(n=1,720$ patients). Classification based on tumor type included melanoma ( $n=1$ study) and NSCLC $(n=1)$. Classification according to the use of ICIs included pembrolizumab $(n=1)$ and durvalumab $(n=1)$. The pooled RR of all-grade diarrhea incidence was not significant after PD-1/PD-L1 inhibitor monotherapy (RR 1.07, 95\%CI: 0.87-1.32, P=0.516) (Figure 2A).

PD-1/PD-L1 inhibitor monotherapy versus chemotherapy

Eleven RCTs [5, 7, 11, 12, 18, 44-49] compared PD-1/PD-L1 inhibitor monotherapy and conventional chemotherapy $(n=5,915$ patients $)$. Classification according to tumor type included melanoma $(n=3$ study), HNSCC $(n=1)$, Gastric cancer $(n=1)$ and NSCLC $(n=6)$. Classification according to the use of ICIs included nivolumab ( $\mathrm{n}=7$ study), pembrolizumab $(n=3)$ and Avelumab $(n=1)$. The risk of allgrade diarrhea after PD-1/PD-L1 inhibitor monotherapy was significantly decreased (RR $0.69,95 \% \mathrm{CI}$ : 0.49-0.98, P=0.037; Figure 2B). The risk of all-grade diarrhea after PD-1/PD-L1 inhibitor monotherapy in Carcinoma of the Head and Neck and Gastric cancer patients were significantly decreased (RR 0.50, 95\% CI: 0.26-0.98, P=0.043; RR 0.23, 95\% CI: 0.12-0.42, $\mathrm{P}=0.000$ Figure 2B).

\section{PD-1/PD-L1 inhibitor monotherapy versus ipilimumab}

Three RCTs [13, 50, 51] compared PD-1/PD-L1 inhibitor monotherapy and ipilimumab $(n=2,596$ patients). The tumor type was malignant melanoma. Classification according to the use of ICIs was nivolumab $(n=2)$ and pembrolizumab $(n=1)$. The study group of Caroline Robert 2015[13] was divided into two subgroups according to drug intervals and thus can be analyzed as two studies. The pooled RR of all-grade diarrhea incidence after the PD-1/PD-L1 inhibitor monotherapy (nivolumab or pembrolizumab) was significantly decreased (RR $0.60,95 \% \mathrm{CI}$ : 0.53-0.68, P=0.000; Figure 2C).

Nivolumab plus ipilimumab compared to nivolumab monotherapy

Four RCTs [42, 48, 50, 52] compared nivolumab plus ipilimumab and nivolumab monotherapy ( $\mathrm{n}=$ 1,805 patients). Classification of tumor type was SCLC $(n=1$ study), NSCLC $(n=1)$ and melanoma $(n=2)$. Our meta-analysis reveals that nivolumab plus ipilimumab significantly increased the risk of all-grade diarrhea compared to nivolumab monotherapy (RR 1.90, 95\% CI: 1.58-2.30, P=0.000; Figure 2D). 


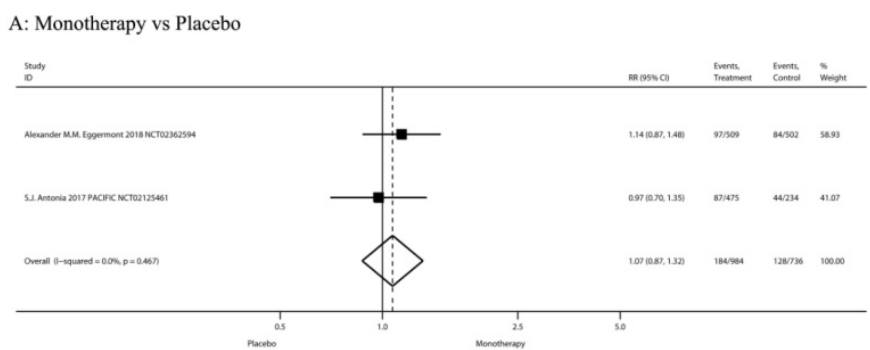

B: Monotherapy vs Chemotherapy
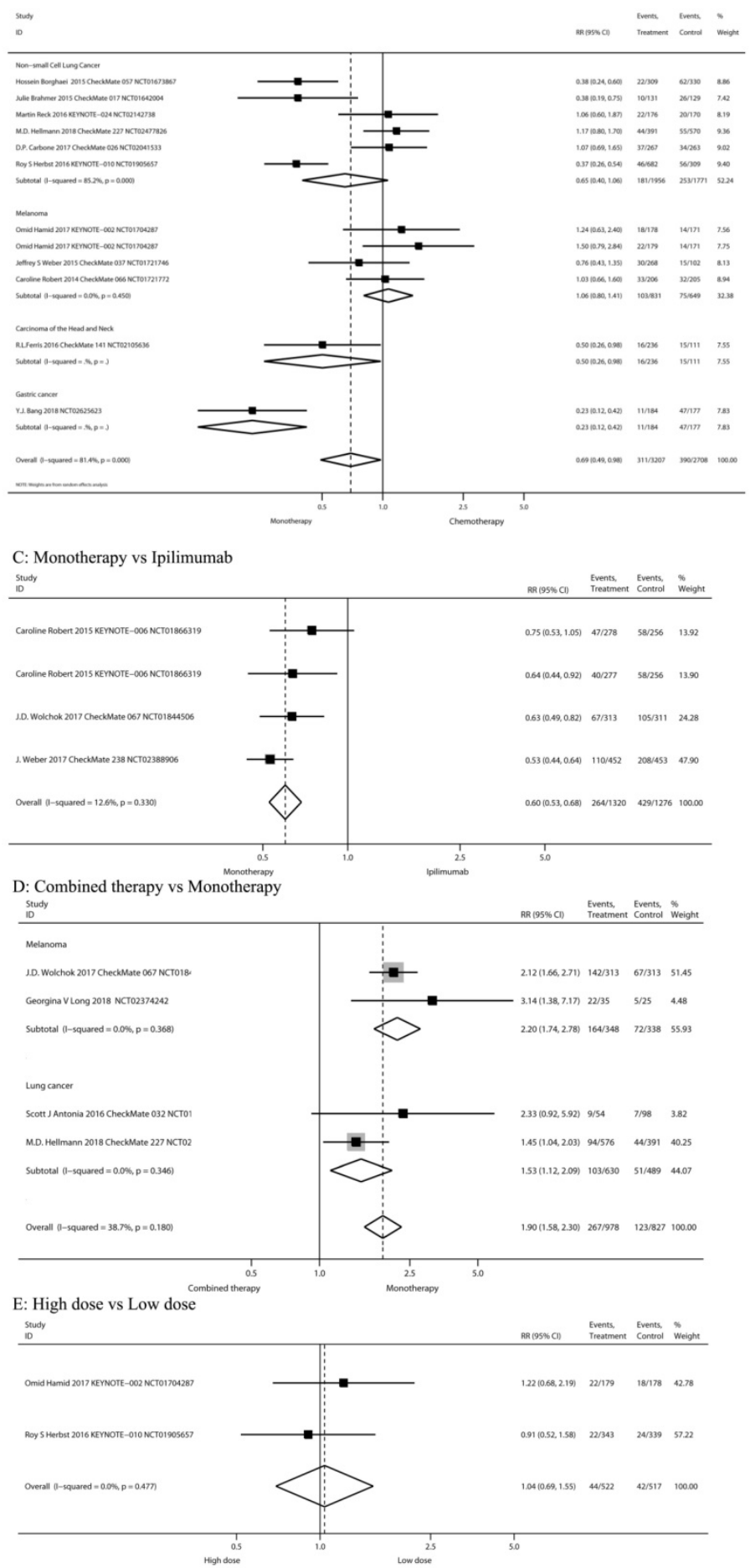

Figure 2. The risks of all-grade diarrhea in PD-1/PD-L1 inhibitors were compared among different treatment regimens. 
Table 1. Characteristics of Relevant Studies

\begin{tabular}{|c|c|c|c|c|c|c|c|c|c|}
\hline $\begin{array}{l}\text { Analysis } \\
\text { Method }\end{array}$ & Source & Format & Data Set & $\begin{array}{l}\text { Tumor } \\
\text { Type }\end{array}$ & Main Inclusion Criterion & Treatment & $\begin{array}{l}\text { Sample } \\
\text { Size }\end{array}$ & $\begin{array}{l}\text { Age, } \\
\text { Median(range), } \\
\text { y }\end{array}$ & $\begin{array}{l}\text { Jadad } \\
\text { score }\end{array}$ \\
\hline \multirow[t]{13}{*}{ Meta-analysis } & $\begin{array}{l}\text { R.L.Ferris } 2016 \\
\text { CheckMate } 141 \\
\text { NCT02105636 }\end{array}$ & Full text & $\begin{array}{l}\text { Randomized, } \\
\text { open-label, } \\
\text { phase } 3 \text { trial }\end{array}$ & $\begin{array}{l}\text { Head and } \\
\text { neck }\end{array}$ & $\begin{array}{l}\text { Recurrent squamous-cell } \\
\text { carcinoma of the head and } \\
\text { neck }\end{array}$ & $\begin{array}{l}\text { 1. Nivolumab } 3 \mathrm{mg} / \mathrm{kg} \text { Q2W } \\
\text { 2. Standard therapy }\end{array}$ & $\begin{array}{l}240 \\
121\end{array}$ & $\begin{array}{l}59(29-83) \\
61(28-78)\end{array}$ & 3 \\
\hline & $\begin{array}{l}\text { Julie Brahmer } \\
2015 \text { CheckMate } \\
017 \text { NCT01642004 }\end{array}$ & Full text & $\begin{array}{l}\text { Randomized, } \\
\text { open-label, } \\
\text { international, } \\
\text { phase } 3 \text { study }\end{array}$ & NSCLC & $\begin{array}{l}\text { Stage IIIB or IV } \\
\text { squamous-cell NSCLC }\end{array}$ & $\begin{array}{l}\text { 1. Nivolumab } 3 \mathrm{mg} / \mathrm{kg} \text { Q2W } \\
\text { 2. Docetaxel } 75 \mathrm{mg} / \mathrm{m} 2 \text { Q3W }\end{array}$ & $\begin{array}{l}135 \\
137\end{array}$ & $\begin{array}{l}62(39-85) \\
64(42-84)\end{array}$ & 3 \\
\hline & $\begin{array}{l}\text { Caroline Robert } \\
2015 \\
\text { KEYNOTE-006 } \\
\text { NCT01866319 }\end{array}$ & Full text & $\begin{array}{l}\text { Randomized, } \\
\text { controlled, } \\
\text { phase } 3 \text { study }\end{array}$ & Melanoma & $\begin{array}{l}\text { Unresectable stage III or IV } \\
\text { melanoma }\end{array}$ & $\begin{array}{l}\text { 1. Pembrolizumab } 10 \mathrm{mg} / \mathrm{kg} \\
\text { Q2W } \\
\text { 2. Pembrolizumab } 10 \mathrm{mg} / \mathrm{kg} \\
\text { Q3W } \\
\text { 3. Ipilimumab } 3 \mathrm{mg} / \mathrm{kg} \mathrm{Q3W}\end{array}$ & $\begin{array}{l}279 \\
277 \\
278\end{array}$ & $\begin{array}{l}61(18-89) \\
63(22-89) \\
62(18-88)\end{array}$ & 3 \\
\hline & $\begin{array}{l}\text { Omid Hamid } \\
2017 \\
\text { KEYNOTE-002 } \\
\text { NCT01704287 }\end{array}$ & Full text & $\begin{array}{l}\text { Randomised, } \\
\text { open-label, } \\
\text { phase } 2 \text { study }\end{array}$ & Melanoma & $\begin{array}{l}\text { Unresectable stage III or } \\
\text { stage IV melanoma not } \\
\text { amenable to local therapy }\end{array}$ & $\begin{array}{l}\text { 1. Pembrolizumab } 2 \mathrm{mg} / \mathrm{kg} \\
\text { Q3W } \\
\text { 2. Pembrolizumab } 10 \mathrm{mg} / \mathrm{kg} \\
\text { Q3W } \\
\text { 3. Chemotherapy(carboplatin } \\
\text {, carboplatin plus paclitaxel, } \\
\text { dacarbazine, paclitaxel alone } \\
\text { or oral temozolomide) }\end{array}$ & $\begin{array}{l}180 \\
181 \\
179\end{array}$ & $\begin{array}{l}62(15-87) \\
60(27-89) \\
63(27-87)\end{array}$ & 3 \\
\hline & $\begin{array}{l}\text { J.D. Wolchok } 2017 \\
\text { CheckMate } 067 \\
\text { NCT01844505 }\end{array}$ & Full text & $\begin{array}{l}\text { Double-blind, } \\
\text { Randomised, } \\
\text { phase } 3 \text { trial }\end{array}$ & Melanoma & $\begin{array}{l}\text { Stage III (unresectable) or } \\
\text { stage IV melanoma }\end{array}$ & $\begin{array}{l}\text { 1.Nivolumab } 1 \mathrm{mg} / \mathrm{kg} \text { Q3W } \\
\text { plus ipilimumab } 3 \mathrm{mg} / \mathrm{kg} \\
\text { Q3W for four doses, followed } \\
\text { by nivolumab 3mg/ } / \mathrm{kg} \mathrm{Q2W} \\
\text { 2.Nivolumab } 3 \mathrm{mg} / \mathrm{kg} \mathrm{Q2W} \\
\text { 3.Ipilimumab } 3 \mathrm{mg} / \mathrm{kg} \mathrm{Q3W} \\
\text { for four doses }\end{array}$ & $\begin{array}{l}314 \\
316 \\
315\end{array}$ & $\begin{array}{l}61(18-88) \\
60(25-90) \\
62(18-89)\end{array}$ & 4 \\
\hline & $\begin{array}{l}\text { Michael A. } \\
\text { Postow } \\
\text { 2015NCT0192741 } \\
9\end{array}$ & Full text & $\begin{array}{l}\text { Randomized } \\
2: 1 \text { in a } \\
\text { double-blinded } \\
\text { phase } 2 \text { trial }\end{array}$ & Melanoma & $\begin{array}{l}\text { Unresectable, } \\
\text { previously-untreated, stage } \\
\text { III or IV melanoma with } \\
\text { measurable disease }\end{array}$ & $\begin{array}{l}\text { 1. Nivolumab } 1 \mathrm{mg} / \mathrm{kg} \text { Q3W } \\
\text { plus ipilimumab } 3 \mathrm{mg} / \mathrm{kg} \text { first } \\
4 \text { doses, then nivolumab } \\
3 \mathrm{mg} / \mathrm{kg} \text { Q2W } \\
\text { 2.Ipilimumab } 3 \mathrm{mg} / \mathrm{kg} \text { first } 4 \\
\text { doses }\end{array}$ & $\begin{array}{l}95 \\
47\end{array}$ & $\begin{array}{l}64(27-87) \\
67(31-80)\end{array}$ & 4 \\
\hline & $\begin{array}{l}\text { Georgina V Long } \\
2018 \text { NCT0237424 } \\
2\end{array}$ & Full text & $\begin{array}{l}\text { Multicentre, } \\
\text { open-label } \\
\text { randomised, } \\
\text { phase } 2 \text { trial }\end{array}$ & Melanoma & Melanoma brain metastases & $\begin{array}{l}\text { 1. Nivolumab } 1 \mathrm{mg} / \mathrm{kg}+ \\
\text { ipilimumab } 3 \mathrm{mg} / \mathrm{kg} \mathrm{Q} 3 \mathrm{~W} \text { for } \\
\text { four doses; then nivolumab } 3 \\
\mathrm{mg} / \mathrm{kg} \mathrm{Q} 2 \mathrm{~W} \\
\text { 2. Nivolumab } 3 \mathrm{mg} / \mathrm{kg} \mathrm{Q} 2 \mathrm{~W} \\
\text { 3. Nivolumab } 3 \mathrm{mg} / \mathrm{kg} \mathrm{Q} 2 \mathrm{~W}( \\
\text { brain metastases) }\end{array}$ & $\begin{array}{l}35 \\
25 \\
16\end{array}$ & $\begin{array}{l}59(53-68) \\
63(52-74) \\
51(48-56)\end{array}$ & 3 \\
\hline & $\begin{array}{l}\text { Caroline Robert, } \\
\text { 2014KEYNOTE-0 } \\
01 \text { NCT01295827 }\end{array}$ & Full text & $\begin{array}{l}\text { Open-label, } \\
\text { international, } \\
\text { multicentre } \\
\text { phase } 1 \text { trial }\end{array}$ & Melanoma & $\begin{array}{l}\text { Progressive, measurable, } \\
\text { unresectable melanoma }\end{array}$ & $\begin{array}{l}\text { 1.Pembrolizumab } 2 \mathrm{mg} / \mathrm{kg} \\
\text { Q3W } \\
\text { 2.Pembrolizumab } 10 \mathrm{mg} / \mathrm{kg} \\
\text { Q3W }\end{array}$ & $\begin{array}{l}89 \\
84\end{array}$ & $\begin{array}{l}57(18-88) \\
60.7(27-86)\end{array}$ & 3 \\
\hline & $\begin{array}{l}\text { Alexander M.M. } \\
\text { Eggermont } 2018 \\
\text { NCT02362594 }\end{array}$ & Full text & $\begin{array}{l}\text { Randomized, } \\
\text { double-blind } \\
\text { phase } 3 \text { trial }\end{array}$ & Melanoma & Resected stage III melanoma & $\begin{array}{l}\text { 1. Pembrolizumab } 200 \mathrm{mg} \\
\text { Q3W for } 18 \text { doses } \\
\text { 2. Placebo }\end{array}$ & $\begin{array}{l}514 \\
505\end{array}$ & $\begin{array}{l}54(19-88) \\
54(19-83)\end{array}$ & 4 \\
\hline & $\begin{array}{l}\text { S.J.Antonia } 2017 \\
\text { PACIFIC } \\
\text { NCT02125461 }\end{array}$ & Full text & $\begin{array}{l}\text { Global, } \\
\text { randomized, } \\
\text { phase } 3 \text { trial }\end{array}$ & NSCLC & $\begin{array}{l}\text { Stage III, locally advanced, } \\
\text { unresectable } \\
\text { NSCLC }\end{array}$ & $\begin{array}{l}\text { 1. Durvalumab10 } \mathrm{mg} / \mathrm{kg} \text { Q2W } \\
\text { for up to } 12 \text { months. } \\
\text { 2. Placebo }\end{array}$ & $\begin{array}{l}476 \\
237\end{array}$ & $\begin{array}{l}64(31-84) \\
64(23-90)\end{array}$ & 3 \\
\hline & $\begin{array}{l}\text { Martin Reck } 2016 \\
\text { KEYNOTE-024 } \\
\text { NCT02142738 }\end{array}$ & Full text & $\begin{array}{l}\text { Open-label, } \\
\text { randomised, } \\
\text { phase } 3 \text { trial }\end{array}$ & NSCLC & $\begin{array}{l}\text { Untreated advanced } \\
\text { NSCLC with PD-L1 } \\
\text { expression on at least } 50 \% \\
\text { of tumor cells kinase gene }\end{array}$ & $\begin{array}{l}\text { 1. Pembrolizumab } 200 \mathrm{mg} \\
\text { Q3W for } 35 \text { cycles } \\
\text { 2.Investigator's choice of } \\
\text { platinum based chemotherapy } \\
\text { for } 4 \text { to } 6 \text { cycles }\end{array}$ & $\begin{array}{l}154 \\
151\end{array}$ & $\begin{array}{l}64.5(33-90) \\
66(38-85)\end{array}$ & 3 \\
\hline & $\begin{array}{l}\text { D.P. Carbone } \\
2017 \text { CheckMate } \\
026 \text { NCT02041533 }\end{array}$ & Full text & $\begin{array}{l}\text { Open-label } \\
\text { randomized } \\
\text { phase } 3 \text { trial }\end{array}$ & NSCLC & $\begin{array}{l}\text { Untreated stage IV or } \\
\text { recurrent NSCLC and a } \\
\text { PD-L1 tumor-expression } \\
\text { level of } 1 \% \text { or more }\end{array}$ & $\begin{array}{l}\text { 1. Nivolumab } 3 \mathrm{mg} / \mathrm{kg} \text { Q2W } \\
\text { 2. Platinum-based } \\
\text { chemotherapy Q3W for up to } \\
\text { six cycles }\end{array}$ & $\begin{array}{l}271 \\
270\end{array}$ & $\begin{array}{l}63(32-89) \\
65(29-87)\end{array}$ & 3 \\
\hline & $\begin{array}{l}\text { Roy S Herbst } 2016 \\
\text { KEYNOTE-010 } \\
\text { NCT01905657 }\end{array}$ & Full text & $\begin{array}{l}\text { Randomised, } \\
\text { open-label, } \\
\text { phase } 2 / 3 \\
\text { study }\end{array}$ & NSCLC & $\begin{array}{l}\text { Previously treated } \\
\text { non-small-cell lung cancer } \\
\text { with PD-L1 expression on at } \\
\text { least } 1 \% \text { of tumour cells }\end{array}$ & $\begin{array}{l}\text { 1. Pembrolizumab } 2 \mathrm{mg} / \mathrm{kg} \\
\text { Q3W } \\
\text { 2. Pembrolizumab } 10 \mathrm{mg} / \mathrm{kg} \\
\text { Q3W } \\
\text { 3. Docetaxel } 75 \mathrm{mg} / \mathrm{m}^{2} \mathrm{Q} 3 \mathrm{~W}\end{array}$ & $\begin{array}{l}344 \\
346 \\
343\end{array}$ & $\begin{array}{l}63(56-69) \\
63(56-69) \\
62(56-69)\end{array}$ & 3 \\
\hline \multirow{2}{*}{ Meta-analysis } & $\begin{array}{l}\text { Scott J Antonia } \\
\text { 2016 CheckMate } \\
\text { 032 NCT01928394 }\end{array}$ & Full text & $\begin{array}{l}\text { Randomised,Co } \\
\text { hort of this } \\
\text { phase } 1 / 2 \\
\text { multicentre, } \\
\text { multi-arm, } \\
\text { open-label trial }\end{array}$ & $\begin{array}{l}\text { Recurrent } \\
\text { small-cell } \\
\text { lung } \\
\text { cancer }\end{array}$ & $\begin{array}{l}\text { Limited-stage or } \\
\text { extensive-stage SCLC, and } \\
\text { had disease progression } \\
\text { after at least one previous } \\
\text { platinum-containing } \\
\text { regimen }\end{array}$ & $\begin{array}{l}\text { 1. Nivolumab } 3 \mathrm{mg} / \mathrm{kg} \text { Q2W } \\
\text { 2. Nivolumab } 1 \mathrm{mg} / \mathrm{kg} \text { plus } \\
\text { ipilimumab } 3 \mathrm{mg} / \mathrm{kg} \text { Q3W for } \\
\text { four cycles, nivolumab3 } \\
\mathrm{mg} / \mathrm{kg} \text { Q2W } \\
\text { 3. Nivolumab } 3 \mathrm{mg} / \mathrm{kg} \text { plus } \\
\text { ipilimumab } 1 \mathrm{mg} / \mathrm{kg} \mathrm{Q} 3 W \text { for } \\
\text { four cycles, nivolumab3 } \\
\mathrm{mg} / \mathrm{kg} \mathrm{Q} 2 \mathrm{~W}\end{array}$ & $\begin{array}{l}98 \\
61 \\
54\end{array}$ & $\begin{array}{l}63(57-68) \\
66(58-71) \\
61(56-65)\end{array}$ & 3 \\
\hline & $\begin{array}{l}\text { M.D. Hellmann } \\
2018 \text { CheckMate } \\
227 \text { NCT02477826 }\end{array}$ & Full text & $\begin{array}{l}\text { Randomised,M } \\
\text { ultipart, } \\
\text { open-label } \\
\text { phase } 3 \text { trial }\end{array}$ & $\begin{array}{l}\text { stage IV or } \\
\text { recurrent } \\
\text { NSCLC }\end{array}$ & $\begin{array}{l}\text { stage IV or recurrent } \\
\text { NSCLC that was not } \\
\text { previously treated with } \\
\text { chemotherapy }\end{array}$ & $\begin{array}{l}\text { 1. Nivolumab } 3 \mathrm{mg} / \mathrm{kg} \mathrm{Q} 2 \mathrm{~W} \\
\text { plus Ipilimumab } 1 \mathrm{mg} / \mathrm{kg} \\
\text { Q6W } \\
\text { 2. Nivolumab } 240 \mathrm{mg} \mathrm{Q2W}\end{array}$ & $\begin{array}{l}576 \\
391 \\
570\end{array}$ & - & 3 \\
\hline
\end{tabular}




\begin{tabular}{|c|c|c|c|c|c|c|c|c|c|}
\hline \multirow[t]{8}{*}{$\begin{array}{l}\text { Analysis } \\
\text { Method }\end{array}$} & Source & Format & Data Set & $\begin{array}{l}\text { Tumor } \\
\text { Type }\end{array}$ & Main Inclusion Criterion & Treatment & $\begin{array}{l}\text { Sample } \\
\text { Size }\end{array}$ & $\begin{array}{l}\text { Age, } \\
\text { Median(range), } \\
\text { y }\end{array}$ & $\begin{array}{l}\text { Jadad } \\
\text { score }\end{array}$ \\
\hline & & & & & & 3. Chemotherapy & & & \\
\hline & $\begin{array}{l}\text { Hossein Borghaei } \\
2015 \text { CheckMate } \\
\text { 057 NCT01673867 }\end{array}$ & Full text & $\begin{array}{l}\text { Randomised, } \\
\text { phase } 3 \text { study }\end{array}$ & $\begin{array}{l}\text { Non-squa } \\
\text { mous } \\
\text { NSCLC }\end{array}$ & $\begin{array}{l}\text { stage IIIB/IV or recurrent } \\
\text { non-squamous NSCLC }\end{array}$ & $\begin{array}{l}\text { 1. Nivolumab } 3 \mathrm{mg} / \mathrm{kg} \text { Q2W } \\
\text { 2. Docetaxel } 75 \mathrm{mg} / \mathrm{m} 2 \text { Q3W }\end{array}$ & $\begin{array}{l}292 \\
290\end{array}$ & $\begin{array}{l}61(37-84) \\
64(21-85)\end{array}$ & 3 \\
\hline & $\begin{array}{l}\text { Jeffrey S Weber } \\
2015 \text { CheckMate } \\
037 \text { NCT01721746 }\end{array}$ & Full text & $\begin{array}{l}\text { Randomised, } \\
\text { controlled, } \\
\text { open-label, } \\
\text { phase } 3 \text { trial }\end{array}$ & melanoma & $\begin{array}{l}\text { unresectable or metastatic } \\
\text { melanoma, and progressed } \\
\text { after ipilimumab, or } \\
\text { ipilimumab and a BRAF } \\
\text { inhibitor if they were } \\
B R A F V^{600} \text { mutation-positive }\end{array}$ & $\begin{array}{l}\text { 1. Nivolumab } 3 \mathrm{mg} / \mathrm{kg} \mathrm{Q} 2 \mathrm{~W} \\
\text { 2. ICC(dacarbazine } 1000 \\
\mathrm{mg} / \mathrm{m}^{2} \mathrm{Q} 3 \mathrm{~W} \text {; carboplatin area } \\
\text { under the curve 6+ paclitaxel } \\
\left.175 \mathrm{mg} / \mathrm{m}^{2} \mathrm{Q} 3 \mathrm{~W}\right)\end{array}$ & $\begin{array}{l}272 \\
133\end{array}$ & $\begin{array}{l}59(23-88) \\
62(29-85)\end{array}$ & 3 \\
\hline & $\begin{array}{l}\text { Caroline Robert } \\
2014 \text { CheckMate } \\
066 \text { NCT01721772 }\end{array}$ & Full text & $\begin{array}{l}\text { Randomised, } \\
\text { double-blind, } \\
\text { phase } 3 \text { trial }\end{array}$ & Melanoma & $\begin{array}{l}\text { unresectable, previously } \\
\text { untreated stage III or IV } \\
\text { melanoma without a BRAF } \\
\text { mutation }\end{array}$ & $\begin{array}{l}\text { 1. Nivolumab(3 mg/kg Q2W)+ } \\
\text { placebo Q3W } \\
\text { 2. Dacarbazine }(1000 \mathrm{mg} / \mathrm{m} 2 \\
\text { Q3W)+ placebo Q2W }\end{array}$ & $\begin{array}{l}210 \\
208\end{array}$ & $\begin{array}{l}64(18-86) \\
66(26-87)\end{array}$ & 4 \\
\hline & $\begin{array}{l}\text { J. Weber } 2017 \\
\text { CheckMate } 238 \\
\text { NCT02388906 }\end{array}$ & Full text & $\begin{array}{l}\text { Randomized, } \\
\text { double-blind, } \\
\text { phase } 3 \text { trial }\end{array}$ & Melanoma & $\begin{array}{l}\text { complete resection of stage } \\
\text { IIIB,IIIC, or IV melanoma }\end{array}$ & $\begin{array}{l}\text { 1. Nivolumab } 3 \mathrm{mg} / \mathrm{kg} \mathrm{Q} 2 \mathrm{~W} \\
\text { 2. Ipilimumab } 10 \mathrm{mg} / \mathrm{kg} \text { QWW } \\
\text { for four doses }\end{array}$ & $\begin{array}{l}453 \\
453\end{array}$ & $\begin{array}{l}56(19-83) \\
54(18-86)\end{array}$ & 4 \\
\hline & $\begin{array}{l}\text { Robert J. Motzer } \\
2015 \\
\text { NCT01354431 }\end{array}$ & Full text & $\begin{array}{l}\text { Blinded, } \\
\text { randomized, } \\
\text { multicenter } \\
\text { phase II trial }\end{array}$ & $\begin{array}{l}\text { Renal Cell } \\
\text { Carcinoma }\end{array}$ & $\begin{array}{l}\text { Metastatic Renal Cell } \\
\text { Carcinoma }\end{array}$ & $\begin{array}{l}\text { 1. Nivolumab } 0.3 \mathrm{mg} / \mathrm{kg} \text { Q3W } \\
\text { 2. Nivolumab } 2 \mathrm{mg} / \mathrm{kg} \text { Q3W } \\
\text { 3. Nivolumab } 10 \mathrm{mg} / \mathrm{kg} \text { Q3W }\end{array}$ & $\begin{array}{l}60 \\
54 \\
54\end{array}$ & $\begin{array}{l}61 \pm 9 \\
61 \pm 8 \\
61 \pm 10\end{array}$ & 3 \\
\hline & $\begin{array}{l}\text { Y.-J. Bang } 2018 \\
\text { NCT02625623 }\end{array}$ & Full text & $\begin{array}{l}\text { multicentre, } \\
\text { international, } \\
\text { randomised, } \\
\text { open-label, } \\
\text { phase III trial }\end{array}$ & $\begin{array}{l}\text { gastric } \\
\text { cancer }\end{array}$ & $\begin{array}{l}\text { metastatic gastric } \\
\text { cancer/gastrooesophageal } \\
\text { junction cancer }\end{array}$ & $\begin{array}{l}\text { 1. Avelumab } 10 \mathrm{mg} / \mathrm{kg} \text { Q2W } \\
\text { 2. Physician's choice of } \\
\text { chemotherapy }\end{array}$ & $\begin{array}{l}185 \\
186\end{array}$ & $\begin{array}{l}59(29-86) \\
61(18-82)\end{array}$ & 3 \\
\hline
\end{tabular}

\section{High-dose group versus low-dose group}

Three RCTs [7, 26, 47] compared the low-dose and high-dose PD-1/PD-L1 inhibitor monotherapy ( $\mathrm{n}=1039$ patients). Pembrolizumab $2 \mathrm{mg} / \mathrm{kg}$ is defined as low dose; $10 \mathrm{mg} / \mathrm{kg}$ is high-dose. Nivolumab $\leq 2 \mathrm{mg} / \mathrm{kg}$ is defined as low dose, 10 $\mathrm{mg} / \mathrm{kg}$ is high-dose. Classification according to tumor type was melanoma ( $\mathrm{n}=1$ study), $\operatorname{RCC}(\mathrm{n}=1)$ and NSCLC $(n=1)$. Results showed no significant risk in the high-dose group (RR 1.15, 95\%CI: 0.80-1.67, $\mathrm{P}=0.446$; Figure 2E).

\section{High-grade diarrhea}

Figure 3 showed the risk of high-grade $(\geq 3)$ diarrhea according to different treatment regimens: PD-1/PD-L1 inhibitor monotherapy versus PD-1/PD-L1 inhibitor plus ipilimumab, versus chemotherapy, versus placebo, versus ipilimumab, and low-dose versus high-dose.

\section{PD-1/PD-L1 inhibitor monotherapy versus placebo}

Inclusion of the study and number of patient's high-grade diarrhea were consistent with previous all-grade diarrhea. As shown in Figure 3A, when compared with placebo, there was not a significant increase in the risk of high-grade diarrhea incidence for PD-1/PD-L1 inhibitor monotherapy (RR 0.85, 95\%CI: 0.29-2.44, $\mathrm{P}=0.756$ ).

\section{PD-1/PD-L1 inhibitor monotherapy versus chemotherapy}

Inclusion of the study and number of patient's high-grade diarrhea were consistent with previous all-grade diarrhea. Results showed a significant decreased in the risk of high-grade diarrhea after monotherapy (RR 0.50, 95\% CI: 0.26-0.95, P = 0.035; Figure 3B). Of the 7 RCTs on non-small cell lung cancer, 5 were treated with nivolumab and 2 with pembrolizumab. The use of nivolumab or pembrolizumab seems to reduce the risk of diarrhea compared to chemotherapy, but the results are not significant (RR 0.58, 95\%CI: 0.25-1.31, P = 0.190; RR 0.61, 95\% CI: 0.03-13.01, $\mathrm{P}=0.754$, respectively).

\section{PD-1/PD-L1 inhibitor monotherapy versus ipilimumab}

Inclusion of the study and number of patients of high-grade diarrhea were consistent with previous grades of diarrhea. Results showed significantly decreased in the risk of high-grade diarrhea after PD-1/PD-L1 inhibitor monotherapy (RR 0.38, 95\% CI: 0.18-0.79, $\mathrm{P}=0.009$; Figure 3C). Our meta-analysis reveals that PD-1 antibodies (pembrolizumab or nivolumab) reduce the risk of severe diarrhea compared to ipilimumab.

\section{Nivolumab plus ipilimumab compared to nivolumab monotherapy}

Inclusion of the study and number of patients of high-grade diarrhea were consistent with previous grades of diarrhea. The tumor type was melanoma in all cases. Results showed no significant increase in the risk of high-grade diarrhea after nivolumab plus ipilimumab treatment (RR 3.29, 95\%CI: 1.80-6.03, $\mathrm{P}=0.000$; Figure 3E). 


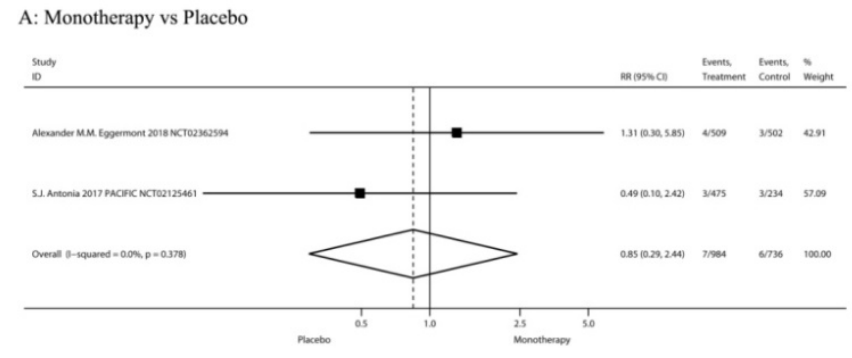

B: Monotherapy vs Chemotherapy

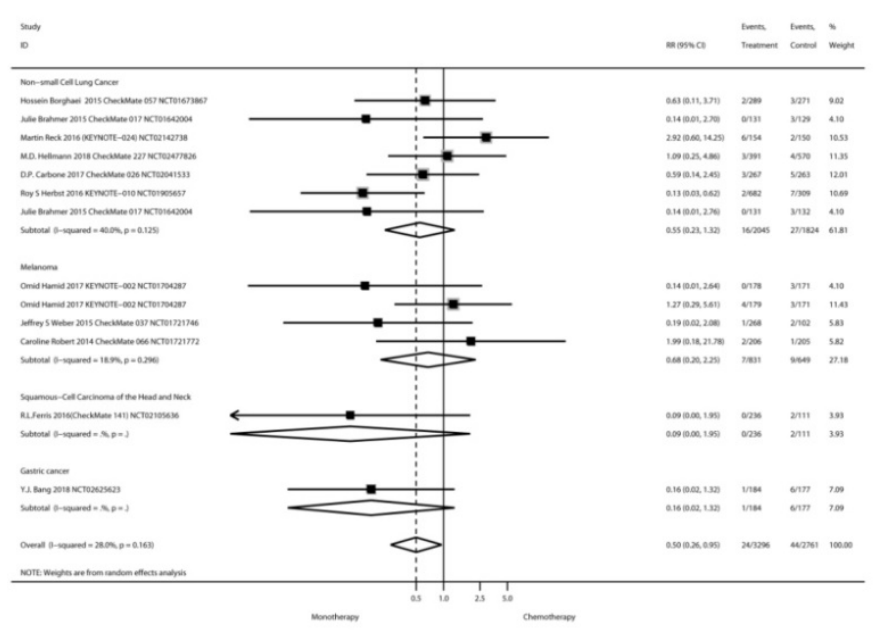

C: Monotherapy vs Ipilimumab

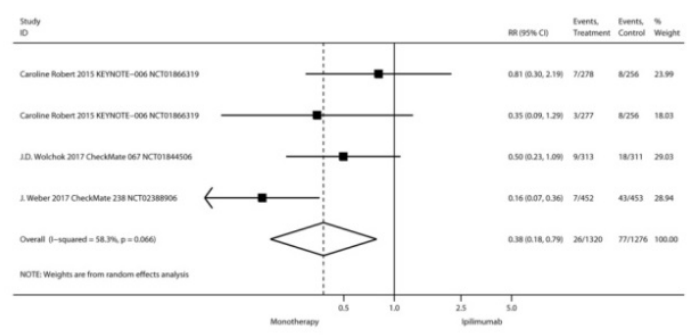

D: Combined therapy vs Monotherapy

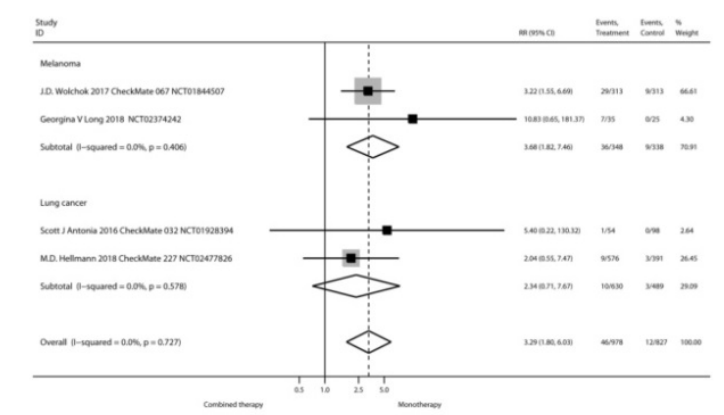

E: High dose vs Low dose

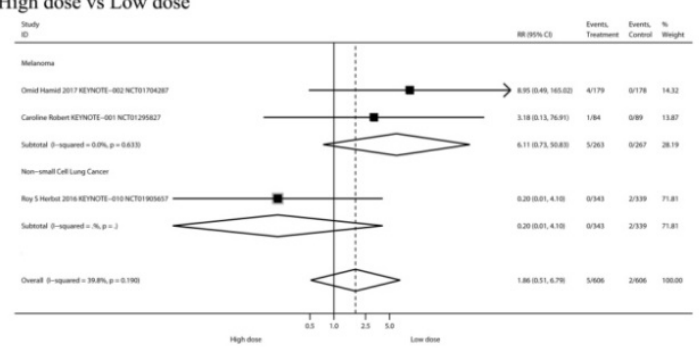

Figure 3. The risk of high-grade $(\geq 3)$ diarrhea according to different treatment regimens.

High-dose group versus low-dose group

Three RCTs [7, 12, 47] compared the High-dose and Low-dose treatments $(n=1,212$ patients). Classification of tumor type was melanoma ( $\mathrm{n}=2$ studies) and NSCLC $(\mathrm{n}=1)$. Results showed no significant increase in the risk of high-grade diarrhea after high-dose treatment (RR 1.86, 95\% CI: 0.51-6.79, P=0.345; Figure 3F).

\section{Study quality and publication bias}

Fifteen trials were open label, whereas five trials were double blind controlled. The Jadad score ranged from 3 to 4 . For RR of all-grade between PD-1/PD-L1 inhibitor monotherapy and chemotherapy or high-grade diarrhea between the monotherapy and ipilimumab, the Egger test suggested some evidence of publication bias. No evidence of bias was found in other comparisons of Egger tests (all $\mathrm{P}>0.05$ ), or in all Begg tests (all $\mathrm{P}>0.05)$.

\section{Discussion}

Although an increasing number of clinical studies have confirmed the overall survival benefit of PD-1/PD-L1 inhibitors treatment, PD-1/PD-L1 inhibitors therapy increases the toxicity of the drug remains controversial, especially diarrhea. Our meta-analysis is the first large-scale analysis of different immunologic treatment regimens compared with chemotherapy or ipilimumab for the toxic side effects of diarrhea. In our research, the risk of all-grade diarrhea after the PD-1/PD-L1 inhibitors plus CTLA- 4 inhibitor combination was 1.90 times higher than that of PD-1/PD-L1 inhibitors monotherapy $(\mathrm{P}<0.05)$, and the risk was 0.72 and 0.60 times higher than that of chemotherapy and ipilimumab compared with monotherapy $(\mathrm{P}<0.05)$. Chemotherapy is the most prone to diarrhea, followed by PD-1/PD-L1 inhibitors plus CTLA-4 inhibitor combination, and finally PD-1/PD-L1 inhibitors monotherapy. When compared with placebo, we did not observe a significant increase in the risk of all-grade or severe diarrhea incidence, and our meta-analysis also reveals that high-dose PD-1/PD-L1 inhibitor monotherapy did not increase the risk of all-grade or severe diarrhea when compared with low-dose(all P>0.05), which suggested that PD-1/PD-L1 inhibitor monotherapy is relatively safe. The risk of grade $\geq 3$ diarrheas for PD-1/PD-L1 inhibitors alone significantly decreased than chemotherapy or ipilimumab, while 
the PD-1/PD-L1 inhibitors plus CTLA-4 inhibitor combination significantly increase than monotherapy.

The basic principle of binding PD-1 / PD-L1 inhibitors and CTLA-4 inhibitors is that they have different mechanisms of action. Anti-CTLA-4 mainly acts on the lymph node area, restores the induction and proliferation of activated T cells, and resists PD-1 acts mainly on the periphery of the tumor site, preventing the tumor-infiltrating tumor-infiltrating PD-L1-expressing tumor and plasma-like dendritic cells from neutralizing cytotoxic $T$ cells [53]. Our result found that patients taking CTLA-4 inhibitors ipilimumab had a significantly higher risk of developing diarrhea than those using PD-1 / PD-L1 inhibitors. This is likely to be related to the different mechanisms of action of the two drugs. Although the combination of PD-1/PD-L1 inhibitors plus CTLA-4 inhibitor has achieved good efficacy [25, 50, 52], the corresponding toxic side effects of combination therapy, especially diarrhea, are significantly higher than those of PD-1/PD-L1 inhibitors alone. Combination therapy with both CTLA-4 and PD-1 blockers raised the risk of GI toxicities to about $45 \%$ which is much higher than monotherapy [54]. The risk of diarrhea was significantly different compared to the use of PD-1/PD-L1 inhibitors alone and chemotherapy in different tumor types. In patients with NSCLC patients, the risk of diarrhea using PD-1 / PD-L1 inhibitors monotherapy is significantly lower than that of patients receiving chemotherapy. However, this result did not find in melanoma patients. Our results show that patients taking pembrolizumab or nivolumab have a slightly different risk of all-grade diarrhea compared with chemotherapy [11, 12, 47]. Furthermore, our study demonstrates no significant difference in the incidence of diarrhea between low-dose versus high-dose PD-1/PD-L1 inhibitors, which are consistent with another study [12]. This provides reliable evidence for further exploration of adjusting drug doses in future clinical trial design and clinical practice.

As far as we know, this is the systematic review including the largest number of RCTs for analysis of immune-related diarrhea. The present study has some limitations. Firstly, these relevant studies have applied PD-1/PD-L1 inhibitors to different treatment lines, and there may be inconsistencies in the underlying characteristics of the patients. Secondly, since the present study is based on a secondary analysis of the final results of each report, we were unable to obtain patient-level disease characteristics and variables, or to determine the specific risk factors associated with the development of immune-related diarrhea. Thirdly, our results were influenced by the limitations of individual clinical trial design. Some of the clinical trials included in the meta- analysis were open label, which may lead to subjective bias. Finally, clinical RCTs included in the meta-analysis had strict inclusion and exclusion criteria. The patients selected in the study were with good PS, but in clinical practice, a large number of patients suffered impaired organ dysfunction and/or functional status and may have a higher incidence of actual toxicity. In the future, large-sample RCTs are needed to compare the incidence and severity of PD-1/PD-L1 inhibitor associated diarrhea among more tumor types and among more combination regimens.

Overall, PD-1/PD-L1 inhibitors have a lower risk of developing diarrhea than chemotherapy and CTLA-4 inhibitor. There is no direct relationship between the dose of PD-1/PD-L1 inhibitors and the risk of developing diarrhea. This study provides reliable evidence for further exploring the combination of PD-1/PD-L1 inhibitors with other drugs in clinical trial design and clinical practice.

\section{Acknowledgements}

\section{Funding \& Support}

This work was supported by grants from the Beijing Natural Science Foundation (grant number 7184200 to Lei Zhao); Beijing Municipal Administration of Hospitals' Youth Programme (grant number QML20170102 to Lei Zhao); Beijing Municipal Administration of Hospitals' Digestive Medical Coordinated Development Center Funding Suppor (grant number XXT01 to Bangwei Cao); Beijing Natural Science Foundation (grant number 7172061 to Bangwei Cao); the Capital Health Research and Development of Special (grant number 2018-2-2022 to Bangwei Cao); China Population Publicity and Education Center Research and Promotion Project (grant number 2017-A001 to Bangwei Cao).

\section{Competing Interests}

The authors have declared that no competing interest exists.

\section{References}

1. Brahmer JR, Drake CG, Wollner I, Powderly JD, Picus J, Sharfman WH et al. Phase I study of single-agent anti-programmed death-1 (MDX-1106) in refractory solid tumors: safety, clinical activity, pharmacodynamics, and immunologic correlates. J Clin Oncol 2010; 28: 3167-3175.

2. Hamid O, Robert C, Daud A, Hodi FS, Hwu WJ, Kefford R et al. Safety and tumor responses with lambrolizumab (anti-PD-1) in melanoma. N Engl J Med 2013; 369: 134-144.

3. Topalian SL, Hodi FS, Brahmer JR, Gettinger SN, Smith DC, McDermott DF et al. Safety, activity, and immune correlates of anti-PD-1 antibody in cancer. $\mathrm{N}$ Engl J Med 2012; 366: 2443-2454.

4. Topalian SL, Sznol M, McDermott DF, Kluger HM, Carvajal RD, Sharfman $\mathrm{WH}$ et al. Survival, durable tumor remission, and long-term safety in patients with advanced melanoma receiving nivolumab. J Clin Oncol 2014; 32: 1020-1030. 
5. Brahmer J, Reckamp KL, Baas P, Crino L, Eberhardt WE, Poddubskaya E et al. Nivolumab versus Docetaxel in Advanced Squamous-Cell Non-Small-Cell Lung Cancer. N Engl J Med 2015; 373: 123-135.

6. Fehrenbacher L, Spira A, Ballinger M, Kowanetz M, Vansteenkiste J, Mazieres $\mathrm{J}$ et al. Atezolizumab versus docetaxel for patients with previously treated non-small-cell lung cancer (POPLAR): a multicentre, open-label, phase 2 randomised controlled trial. Lancet 2016; 387: 1837-1846.

7. Herbst RS, Baas P, Kim DW, Felip E, Perez-Gracia JL, Han JY et al. Pembrolizumab versus docetaxel for previously treated, PD-L1-positive, advanced non-small-cell lung cancer (KEYNOTE-010): a randomised controlled trial. Lancet 2016; 387: 1540-1550.

8. Horn L, Spigel DR, Vokes EE, Holgado E, Ready N, Steins M et al. Nivolumab Versus Docetaxel in Previously Treated Patients With Advanced Non-Small-Cell Lung Cancer: Two-Year Outcomes From Two Randomized, Open-Label, Phase III Trials (CheckMate 017 and CheckMate 057). J Clin Oncol 2017; 35: 3924-3933.

9. Ribas A, Puzanov I, Dummer R, Schadendorf D, Hamid O, Robert C et al. Pembrolizumab versus investigator-choice chemotherapy for ipilimumab-refractory melanoma (KEYNOTE-002): a randomised, controlled, phase 2 trial. Lancet Oncol 2015; 16: 908-918.

10. Robert C, Long GV, Brady B, Dutriaux C, Maio M, Mortier L et al. Nivolumab in previously untreated melanoma without BRAF mutation. N Engl J Med 2015; 372: 320-330.

11. Weber JS, D'Angelo SP, Minor D, Hodi FS, Gutzmer R, Neyns B et al. Nivolumab versus chemotherapy in patients with advanced melanoma who progressed after anti-CTLA-4 treatment (CheckMate 037): a randomised, controlled, open-label, phase 3 trial. Lancet Oncol 2015; 16: 375-384.

12. Robert C, Ribas A, Wolchok JD, Hodi FS, Hamid O, Kefford R et al. Anti-programmed-death-receptor-1 treatment with pembrolizumab in ipilimumab-refractory advanced melanoma: a randomised dose-comparison cohort of a phase 1 trial. Lancet 2014; 384: 1109-1117.

13. Robert C, Schachter J, Long GV, Arance A, Grob JJ, Mortier L et al. Pembrolizumab versus Ipilimumab in Advanced Melanoma. N Engl J Med 2015; 372: 2521-2532

14. Zimmer L, Apuri S, Eroglu Z, Kottschade LA, Forschner A, Gutzmer R et al. Ipilimumab alone or in combination with nivolumab after progression on anti-PD-1 therapy in advanced melanoma. Eur J Cancer 2017; 75: 47-55.

15. Garon EB, Rizvi NA, Hui R, Leighl N, Balmanoukian AS, Eder JP et al. Pembrolizumab for the treatment of non-small-cell lung cancer. N Engl J Med 2015; 372: 2018-2028

16. Hui R, Garon EB, Goldman JW, Leighl NB, Hellmann MD, Patnaik A et al. Pembrolizumab as first-line therapy for patients with PD-L1-positive advanced non-small cell lung cancer: a phase 1 trial. Ann Oncol 2017; 28: 874-881.

17. Langer CJ, Gadgeel SM, Borghaei H, Papadimitrakopoulou VA, Patnaik A, Powell SF et al. Carboplatin and pemetrexed with or without pembrolizumab for advanced, non-squamous non-small-cell lung cancer: a randomised, phase 2 cohort of the open-label KEYNOTE-021 study. Lancet Oncol 2016; 17: 1497-1508.

18. Reck M, Rodriguez-Abreu D, Robinson AG, Hui R, Csoszi T, Fulop A et al. Pembrolizumab versus Chemotherapy for PD-L1-Positive Non-Small-Cell Lung Cancer. N Engl J Med 2016; 375: 1823-1833.

19. Sul J, Blumenthal GM, Jiang X, He K, Keegan P, Pazdur R. FDA Approval Summary: Pembrolizumab for the Treatment of Patients With Metastatic Non-Small Cell Lung Cancer Whose Tumors Express Programmed Death-Ligand 1. Oncologist 2016; 21: 643-650.

20. Seiwert TY, Burtness B, Mehra R, Weiss J, Berger R, Eder JP et al. Safety and clinical activity of pembrolizumab for treatment of recurrent or metastatic squamous cell carcinoma of the head and neck (KEYNOTE-012): an open-label, multicentre, phase $1 \mathrm{~b}$ trial. Lancet Oncol 2016; 17: 956-965.

21. Chen R, Zinzani PL, Fanale MA, Armand P, Johnson NA, Brice P et al. Phase II Study of the Efficacy and Safety of Pembrolizumab for Relapsed/Refractory Classic Hodgkin Lymphoma. J Clin Oncol 2017; 35: 2125-2132.

22. Balar AV, Galsky MD, Rosenberg JE, Powles T, Petrylak DP, Bellmunt J et al. Atezolizumab as first-line treatment in cisplatin-ineligible patients with locally advanced and metastatic urothelial carcinoma: a single-arm, multicentre, phase 2 trial. Lancet 2017; 389: 67-76

23. Bellmunt J, de Wit R, Vaughn DJ, Fradet Y, Lee JL, Fong L et al. Pembrolizumab as Second-Line Therapy for Advanced Urothelial Carcinoma. N Engl J Med 2017; 376: 1015-1026.

24. Fuchs CS, Doi T, Jang RW, Muro K, Satoh T, Machado M et al. Safety and Efficacy of Pembrolizumab Monotherapy in Patients With Previously Treated Advanced Gastric and Gastroesophageal Junction Cancer: Phase 2 Clinical KEYNOTE-059 Trial. JAMA Oncol 2018; 4: e180013.

25. Postow MA, Chesney J, Pavlick AC, Robert C, Grossmann K, McDermott D et al. Nivolumab and ipilimumab versus ipilimumab in untreated melanoma. $\mathrm{N}$ Engl J Med 2015; 372: 2006-2017.

26. Motzer RJ, Escudier B, McDermott DF, George S, Hammers HJ, Srinivas S et al. Nivolumab versus Everolimus in Advanced Renal-Cell Carcinoma. N Engl J Med 2015; 373: 1803-1813.

27. Ansell SM, Lesokhin AM, Borrello I, Halwani A, Scott EC, Gutierrez M et al. PD-1 blockade with nivolumab in relapsed or refractory Hodgkin's lymphoma. N Engl J Med 2015; 372: 311-319.

28. Younes A, Santoro A, Shipp M, Zinzani PL, Timmerman JM, Ansell S et al. Nivolumab for classical Hodgkin's lymphoma after failure of both autologous stem-cell transplantation and brentuximab vedotin: a multicentre, multicohort, single-arm phase 2 trial. Lancet Oncol 2016; 17: 1283-1294.

29. Sharma P, Retz M, Siefker-Radtke A, A B, Necchi A, Bedke J et al. Nivolumab in metastatic urothelial carcinoma after platinum therapy (CheckMate 275): a multicentre, single-arm, phase 2 trial. Lancet Oncol 2017; 18: 312-322.

30. Overman MJ, McDermott R, Leach JL, Lonardi S, Lenz HJ, Morse MA et al. Nivolumab in patients with metastatic DNA mismatch repair-deficient or microsatellite instability-high colorectal cancer (CheckMate 142): an open-label, multicentre, phase 2 study. Lancet Oncol 2017; 18: 1182-1191.

31. El-Khoueiry AB, Sangro B, Yau T, Crocenzi TS, Kudo M, Hsu C et al. Nivolumab in patients with advanced hepatocellular carcinoma (CheckMate 040): an open-label, non-comparative, phase $1 / 2$ dose escalation and expansion trial. Lancet 2017; 389: 2492-2502.

32. Rosenberg JE, Hoffman-Censits J, Powles T, van der Heijden MS, Balar AV, Necchi A et al. Atezolizumab in patients with locally advanced and metastatic urothelial carcinoma who have progressed following treatment with platinum-based chemotherapy: a single-arm, multicentre, phase 2 trial. Lancet 2016; 387: 1909-1920.

33. Rittmeyer A, Barlesi F, Waterkamp D, Park K, Ciardiello F, von Pawel J et al. Atezolizumab versus docetaxel in patients with previously treated non-small-cell lung cancer (OAK): a phase 3, open-label, multicentre randomised controlled trial. Lancet 2017; 389: 255-265.

34. Apolo AB, Infante JR, Balmanoukian A, Patel MR, Wang D, Kelly K et al. Avelumab, an Anti-Programmed Death-Ligand 1 Antibody, In Patients With Refractory Metastatic Urothelial Carcinoma: Results From a Multicenter, Phase Ib Study. J Clin Oncol 2017; 35: 2117-2124.

35. Massard C, Gordon MS, Sharma S, Rafii S, Wainberg ZA, Luke J et al. Safety and Efficacy of Durvalumab (MEDI4736), an Anti-Programmed Cell Death Ligand-1 Immune Checkpoint Inhibitor, in Patients With Advanced Urothelial Bladder Cancer. J Clin Oncol 2016; 34: 3119-3125.

36. Michot JM, Bigenwald C, Champiat S, Collins M, Carbonnel F, Postel-Vinay S et al. Immune-related adverse events with immune checkpoint blockade: a comprehensive review. Eur J Cancer 2016; 54: 139-148.

37. Nishino M, Sholl LM, Hodi FS, Hatabu H, Ramaiya NH. Anti-PD-1-Related Pneumonitis during Cancer Immunotherapy. N Engl J Med 2015; 373: 288-290.

38. Tirumani SH, Ramaiya NH, Keraliya A, Bailey ND, Ott PA, Hodi FS et al. Radiographic Profiling of Immune-Related Adverse Events in Advanced Melanoma Patients Treated with Ipilimumab. Cancer Immunol Res 2015; 3: 1185-1192.

39. Weber JS, Kahler KC, Hauschild A. Management of immune-related adverse events and kinetics of response with ipilimumab. J Clin Oncol 2012; 30: 2691-2697.

40. Champiat S, Lambotte O, Barreau E, Belkhir R, Berdelou A, Carbonnel F et al. Management of immune checkpoint blockade dysimmune toxicities: a collaborative position paper. Ann Oncol 2016; 27: 559-574

41. Jadad AR, Moore RA, Carroll D, Jenkinson C, Reynolds DJ, Gavaghan DJ et al. Assessing the quality of reports of randomized clinical trials: is blinding necessary? Control Clin Trials 1996; 17: 1-12.

42. Antonia SJ, Villegas A, Daniel D, Vicente D, Murakami S, Hui R et al. Durvalumab after Chemoradiotherapy in Stage III Non-Small-Cell Lung Cancer. N Engl J Med 2017; 377: 1919-1929.

43. Eggermont A, Blank CU, Mandala M, Long GV, Atkinson V, Dalle S et al. Adjuvant Pembrolizumab versus Placebo in Resected Stage III Melanoma. N Engl J Med 2018; 378: 1789-1801.

44. Borghaei H, Paz-Ares L, Horn L, Spigel DR, Steins M, Ready NE et al. Nivolumab versus Docetaxel in Advanced Nonsquamous Non-Small-Cell Lung Cancer. N Engl J Med 2015; 373: 1627-1639.

45. Carbone DP, Reck M, Paz-Ares L, Creelan B, Horn L, Steins M et al. First-Line Nivolumab in Stage IV or Recurrent Non-Small-Cell Lung Cancer. N Engl J Med 2017; 376: 2415-2426.

46. Ferris RL, Blumenschein GJ, Fayette J, Guigay J, Colevas AD, Licitra L et al. Nivolumab for Recurrent Squamous-Cell Carcinoma of the Head and Neck. N Engl J Med 2016; 375: 1856-1867.

47. Hamid O, Puzanov I, Dummer R, Schachter J, Daud A, Schadendorf D et al. Final analysis of a randomised trial comparing pembrolizumab versus investigator-choice chemotherapy for ipilimumab-refractory advanced melanoma. Eur J Cancer 2017; 86: 37-45.

48. Hellmann MD, Ciuleanu TE, Pluzanski A, Lee JS, Otterson GA, Audigier-Valette $C$ et al. Nivolumab plus Ipilimumab in Lung Cancer with a High Tumor Mutational Burden. N Engl J Med 2018; 378: 2093-2104.

49. Bang YJ, Ruiz EY, Van Cutsem E, Lee KW, Wyrwicz L, Schenker M et al. Phase III, randomised trial of avelumab versus physician's choice of chemotherapy as third-line treatment of patients with advanced gastric or gastro-oesophageal junction cancer: primary analysis of JAVELIN Gastric 300. Ann Oncol 2018; 29: 2052-2060.

50. Wolchok JD, Chiarion-Sileni V, Gonzalez R, Rutkowski P, Grob JJ, Cowey CL et al. Overall Survival with Combined Nivolumab and Ipilimumab in Advanced Melanoma. N Engl J Med 2017; 377: 1345-1356.

51. Weber J, Mandala M, Del Vecchio M, Gogas HJ, Arance AM, Cowey CL et al. Adjuvant Nivolumab versus Ipilimumab in Resected Stage III or IV Melanoma. N Engl J Med 2017; 377: 1824-1835.

52. Long GV, Atkinson V, Lo S, Sandhu S, Guminski AD, Brown MP et al. Combination nivolumab and ipilimumab or nivolumab alone in melanoma brain metastases: a multicentre randomised phase 2 study. Lancet Oncol 2018; 19: 672-681. 
53. Eggermont AMM, Crittenden $\mathrm{M}$, Wargo J. Combination Immunotherapy Development in Melanoma. Am Soc Clin Oncol Educ Book 2018; 197-207.

54. Larkin J, Chiarion-Sileni V, Gonzalez R, Grob JJ, Cowey CL, Lao CD et al.

Combined Nivolumab and Ipilimumab or Monotherapy in Untreated Melanoma. N Engl J Med 2015; 373: 23-34. 